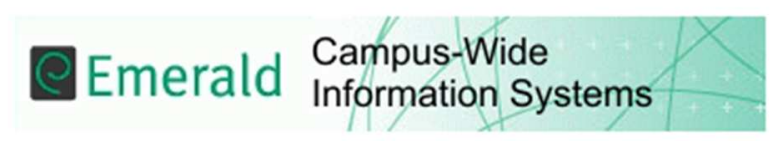

\title{
A Hybrid Approach for Supporting Adaptivity in E-learning Environments
}

\begin{tabular}{|r|l|}
\hline Journal: & International Journal of Information and Learning Technology \\
\hline Manuscript ID & IJILT-04-2016-0014 \\
\hline Manuscript Type: & Research Paper \\
\hline Keywords: & $\begin{array}{l}\text { Learning Management System, Adaptivity, Agent-based System, ECA } \\
\text { model, E-learning, Learning styles }\end{array}$ \\
\hline \multicolumn{2}{|l}{} \\
\hline
\end{tabular}

SCHOLARONE

Manuscripts 


\section{A Hybrid Approach for Supporting Adaptivity in E-learning Environments}

\section{Purpose}

The purpose of this paper is to identify a framework to support adaptivity in e-learning environments. The framework reflects a novel hybrid approach incorporating the concept of the ECA model and intelligent agents. Moreover, a system prototype is developed reflecting the hybrid approach to supporting adaptivity in any given Learning Management System based on learners' learning styles.

\section{Design/methodology/approach}

This paper offers a brief review of current frameworks and systems to support adaptivity in elearning environments. A framework to support adaptivity is designed and discussed, reflecting the hybrid approach in detail. A system prototype is developed incorporating different adaptive features based on the Felder-Silverman learning styles model. Finally, the prototype is implemented in Moodle.

\section{Findings}

The system prototype supports real-time adaptivity in any given Learning Management System based on learners' learning styles. It can deal with any type of content provided by course designers and instructors in the Learning Management System. Moreover, it can support adaptivity at both course and learner levels.

\section{Originality/value}

To the best of our knowledge, no previous work has been done incorporating the concept of the ECA model and intelligent agents as hybrid architecture to support adaptivity in elearning environments. The system prototype has wider applicability and can be adapted to support different types of adaptivity.

\section{Keywords}

Learning Management System, Adaptivity, Agent-based System, ECA model, E-learning, Learning styles 


\section{Introduction}

The Internet and its services have dramatically changed the process of teaching and learning in higher education institutions all over the world. Learning Management Systems (LMSs) and Massive Online Open Courses (MOOCs) are widely used in many educational institutions, such as universities, for the delivery of distance and blended learning. Nevertheless, the applied pedagogical strategies in these systems often do not take into consideration the differences between learners, especially their learning styles. The same LMS courses are presented to all learners on a "one-size-fits-all" basis. This paper presents a novel approach to supporting adaptivity in any given LMS based on learners' learning styles.

The idea of providing adaptive content in LMSs has emerged from Intelligent Tutoring Systems (ITSs) and Adaptive Hyper Media Systems (AHMSs) in which adaptive presentation and navigation are provided (Phobun and Vicheanpanya, 2010). These systems are normally used in computer-based instruction (ibid). There are a considerable number of adaptive learning systems incorporating learning styles such as Arthur (Gilbert and Han, 1999), iWeaver (Wolf, 2003), INSPIRE (Papanikolaou et al., 2003), CS383 (Carver et al., 1999), IDEAL (Shang et al., 2001), and AHA! (Bra et al., 2003). Adaptivity is fully incorporated in most of these systems via the provision of the aforementioned adaptive presentation and navigation. However, these systems lack integration and interoperability. Indeed, according to Brusilovsky (2004), they provide limited functionalities and tools in web educational environments. Currently, these systems are rarely implemented in educational institutions.

In contrast, LMSs such as Moodle, ${ }^{1}$ Blackboard, ${ }^{2}$ and Sakai $^{3}$ are widely used and implemented in many educational institutions worldwide (Pitigala Liyanage et al., 2013). Most of the current LMSs do not support adaptivity: learners' differences such as preferred learning styles were not considered when these systems were designed. E-learning effectiveness can be achieved in LMSs when learners' needs are considered (Xu et al., 2014). Therefore, the motivation behind this paper is primarily to extend the capabilities of LMSs to support adaptivity based on learning styles. In this paper, we show how adaptivity can be achieved in any LMS using a hybrid approach of agent technology and the concept of the Event-Condition-Action (ECA) model.

\footnotetext{
${ }^{1}$ https://moodle.org/

${ }^{2} \mathrm{http}: / /$ blackboard.com

${ }^{3}$ https://www.sakaiproject.org/
} 
The rest of our paper has been divided as follows: Section 2 provides a brief review of related work and introduces the technologies used in the proposed hybrid approach. Section 3 presents a detailed design of our framework reflecting the hybrid approach, and identifies the adaptation features incorporated within it. A prototype is developed and presented to show the applicability of our approach. The last section concludes the paper and states our future work.

\section{Literature Review}

In this part, we provide a brief review of adaptivity in learning systems incorporating learning styles, and we introduce the technologies used in our proposed approach.

\subsection{Learning styles}

There are several learning styles models in existence, which are used by many researchers. Among them, we can mention those developed by Honey and Mumford (1992), Kolb (1984), Dunn and Dunn (Dunn and Griggs, 2003), and Felder-Silverman (Felder and Silverman, 1988). However, the Felder-Silverman Learning Style Model (FSLSM) is one of the most popular models. A study by Al-Azawei and Badii (2014) shows that this model is one of the most dominant and popular learning style models in the literature. This is due to the validity and reliability of its Index of Learning Styles (ILS) (Kolb, 2005; Litzinger et al., 2007; Riding, 1991; Viola et al., 2006). In addition to the suitability and feasibility of this model for technology enhanced learning (Cha et al., 2006; Graf, 2007; Huang et al., 2012; Yang et al., 2013), it is also more consistent with learners' preferences (Villaverde et al., 2006). Thus, the adaptation process proposed in this paper is based on the FSLSM.

\subsection{Agent Technology and the ECA Model}

Recently, there has been an increased interest in implementing agents in the context of elearning systems for supporting adaptivity (Bokhari and Ahmad, 2014; Zhang et al., 2010). There exist several proposed frameworks and systems that incorporate agent technology in order to support adaptivity in e-learning environments. It is impossible to mention all of them, but the work presented in Chang and Chen (2012), Morales-Rodríguez et al. (2012), and Pitigala Liyanage et al. (2013) are worth highlighting. To the best of our knowledge, no work has been done incorporating the concept of the ECA model and agents as a hybrid approach to support adaptivity in e-learning environments. 
The ECA model is widely used in Active Database Management Systems and Workflow Management Systems (Zhi-xue et al., 2012). It comprises three components: event, condition and action (Poulovassilis et al., 2006). Databases are the main repository of the majority of current LMSs. Online activities, such as accessing the system, submitting assignments and accessing courses' content can be seen as events stored in the database of the LMS. Since LMSs take the form of databases where all logs and data about learners and their activities are stored and archived, the ECA model can play a significant role in sensing events in the elearning environment. Indeed, this can be achieved by using database triggers based on predefined pedagogical rules, which can be updated by teachers in the light of new requirements. To achieve our objectives, a proposed framework is designed incorporating agent technology and the concept of the ECA model. Furthermore, using Moodle as the LMS platform, a prototype is developed to reflect the design of the proposed framework and to show how adaptivity is achieved.

\section{The Proposed Framework}

The proposed framework has been previously introduced in Al-Omari et al. (2015). In this paper, the proposed framework, shown in Figure 1, is discussed in more detail to show how it supports adaptivity based on learners' learning styles in e-learning environments. Each component is discussed in order to identify its role in the whole design.

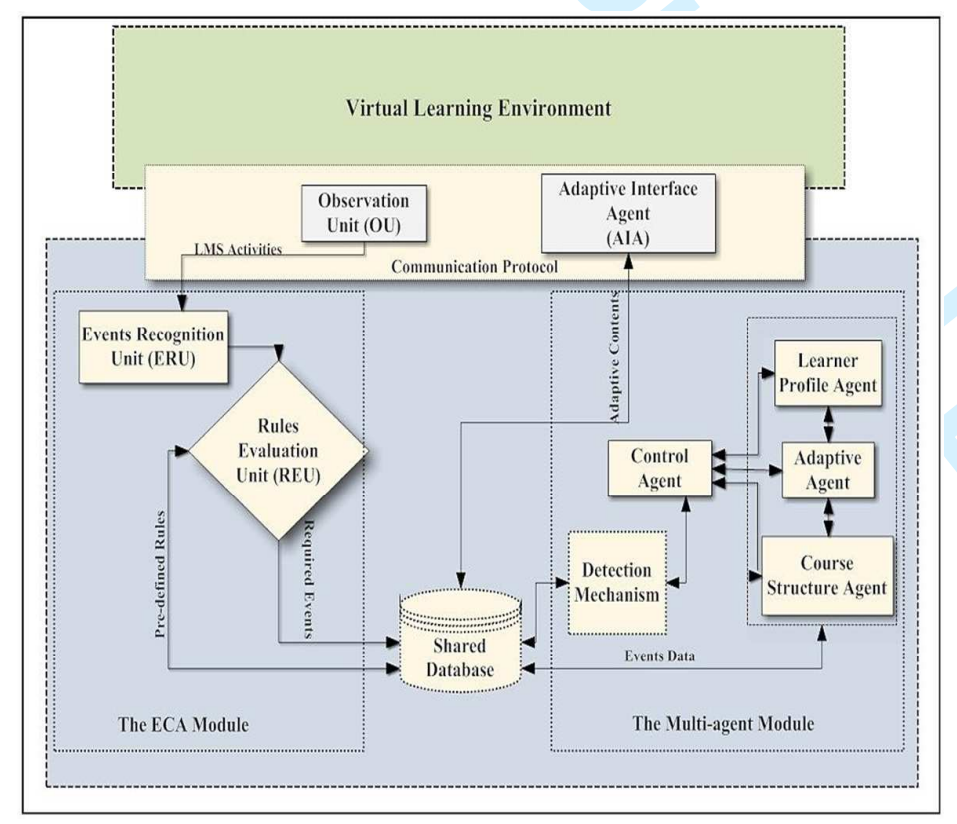

Figure 1: The proposed framework (Al-Omari et al., 2015) 


\subsection{Virtual Learning Environment and an Adaptive Course Structure}

Moodle has been chosen to implement the proposed approach. Moodle is probably the most popular and used platform for LMSs, with over 53,346 sites providing it for over 70 million users across 222 countries (Moodle, 2016). The ILS questionnaire based on the FSLSM (Felder and Soloman, 2005) is implemented in Moodle and designed as a feedback learning activity, as Figure 2 shows. The ILS questionnaire consists of 11 questions for each of the four categories of the FSLSM (a 44-item questionnaire).

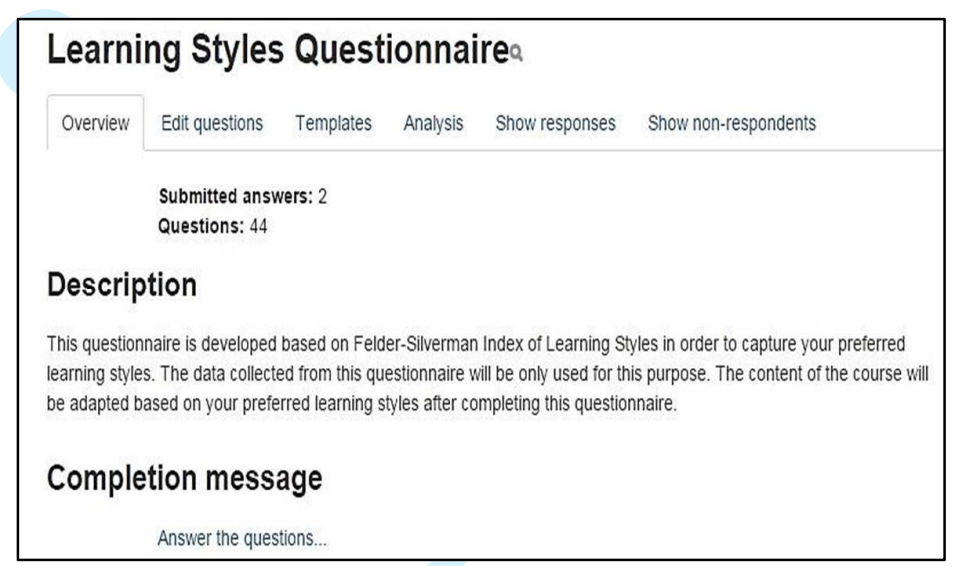

Figure 2: Learning styles questionnaire in Moodle

Courses can be designed with different structures in the LMS depending on the course designers and instructors. However, the course design is based on a specific course structure in the LMS; each course is divided into chapters or sections in which learning resources (e.g. document files, links, videos, etc.) and learning activities (e.g. quizzes, forums, exercises, etc.) are included. Thus, a generic model of an adaptive course structure is shown in Figure 3.

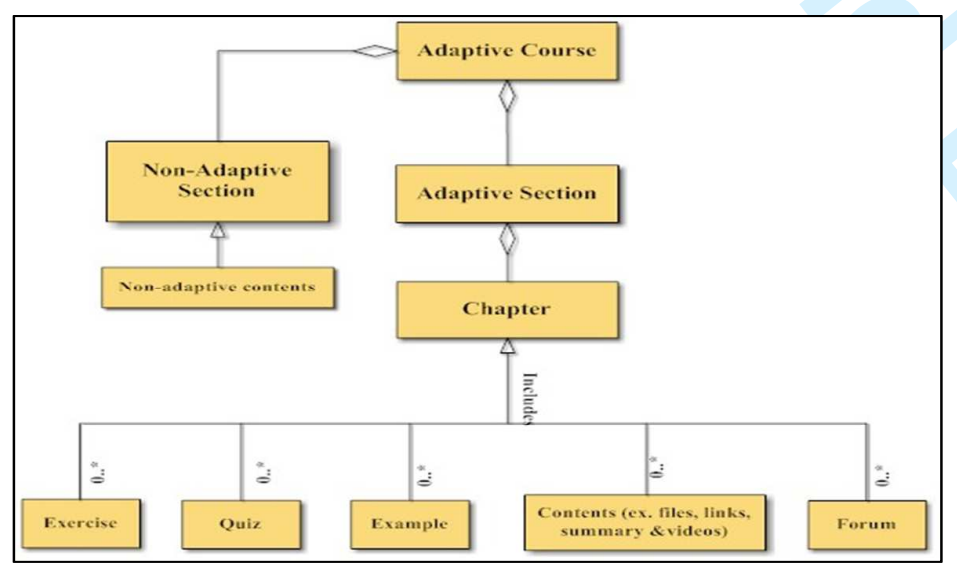

Figure 3: A generic model of an adaptive course structure 
The structure of an adaptive course is designed so that each course consists of sections in which each section reflects a specific chapter. Moreover, the first section in each course is considered as a non-adaptive section where teachers can add any contents that are considered to be required for all learners. For example, teachers can add announcements, online exams, etc. The adaptive sections deal with the actual adaptive contents which are dedicated to each learner.

\subsection{Adaptive Features}

Adaptivity based on learning styles can take the form of adaptive presentation and navigation as the literature suggests. Thus, three main features are integrated in the proposed approach in order to support adaptive presentation and navigation, namely, the type, the number, and the order of the course contents that are recommended to each learner. These adaptive features must comply with each learning style in the FSLSM. It is very important to mention at this level that the primary focus is a system that is able to present different materials according to learning styles. The exact nature of the materials is beyond the scope of this study. Therefore, that part could be offered as future work for researchers interested in pedagogy. The adaptive features are based on Felder and Soloman's (2005) recommendations and discussed according to the four categories of the FSLSM.

\section{- Active/Reflective}

According to Felder and Soloman (2005), working within groups can support active learners in the learning process. They tend to discuss, apply and explain things to others. Moreover, they prefer to learn by actively experimenting. Thus, learning activities such as exercises, quizzes, and discussion forums can help them in the learning process by solving problems and interacting with other learners in the discussion forums. The number of these activities is increased for this type of learner. These learning activities are shown before the learning resources. Since examples can be considered as work accomplished by others, active learners are likely to be uninterested in examples. Thus, the number of examples is reduced for this type of learners. On the other hand, reflective learners are less active in terms of behaviour than active learners. They tend to learn by thinking about the learning material thoroughly and working individually. Therefore, the number of learning activities such as exercises and forums is reduced. Since reflective learners prefer to reflect on the learning material at the beginning of the learning process, exercises and examples are shown after the learning resources. 
- Sensing/Intuitive

Sensing learners prefer to learn from facts that have apparent connections to the real world. Furthermore, they prefer practical work that can reflect the theoretical part of the subject. They like problem-solving activities as well. From this point of view, learning activities such as examples, exercises, and quizzes can support these learners during the learning process. Furthermore, the number of these learning activities is increased for this type of learner. It is recommended that examples are shown before the learning resources whereas exercises and quizzes are shown after. In contrast, intuitive learners prefer challenging work that tests their knowledge. Thus, it is recommended to increase the number of quizzes and show them before the learning resources. As intuitive learners dislike repetition, it is recommended to decrease the number of examples and show them after the learning resources.

\section{- Visual/Verbal}

Visual learners prefer visual contents in order to better understand a particular topic. It is recommended that the contents include several images, figures, charts and videos. Visual learners memorise better when they see objects and as a result can enhance their learning process. Therefore, learning resources which include visual contents are presented to these learners. Conversely, verbal learners prefer textual contents in addition to auditory contents. Visual contents can probably be distracting and confusing for this type of learner. Therefore, these learners are provided with textual contents that suit their preference. In addition, recorded lectures are probably beneficial for this type of learner.

\section{- Sequential/Global}

Sequential learners prefer to learn in a sequential manner, focusing on details and connections between learning topics. It is recommended that learning activities such as exercises and quizzes are presented after or between the learning resources for this type of learner. In contrast, global learners prefer to learn randomly without considering connections between subjects. Therefore, it is recommended to provide this type of learner with outlines before the learning resources in order to help them get the big picture of the learning material. Moreover, exercises and quizzes are recommended to be shown after the material so that these learners can have better knowledge after completing the learning material.

It is very important to mention that the adaptive features mentioned above are considered as 'recommendations' not 'prescriptions' for presenting the contents of an adaptive course in the 
proposed approach. Learners can see the non-adaptive course at any time. The adaptive features are based on a proposed concept of an adaptive course in which suitable learning activities and resources are presented to each learner. It is crucial that course designers and teachers be involved in the process of designing such adaptive courses. They are required to select the suitable learning activities and resources that can support each learner based on his/her learning styles. In order to facilitate this process, the teachers and course designers are provided with a Graphical User Interface (GUI) based on drag and drop facilities.

\subsection{The ECA Module}

All the activities that are performed by the learners are stored in the database of the LMS. As mentioned before, these activities can be seen as events occurring in the database. From this point of view, the completion of the learning style questionnaire is also considered an event occurring in the LMS, which is involved in the adaptation process. The ECA module in the proposed framework is considered as the sensing component which provides the multi-agent module with the required data about LMS events in a timely manner. These data are vital for the adaptation process in order to provide learners with adaptive contents and build learners' models. The ECA module is implemented as a series of database triggers in the database of Moodle. The database triggers can include conditions which should be evaluated before actions are performed. Each component of the ECA module operates as follows:

\section{- The Observation Unit (OU)}

The Observation Unit deals with the required locations (tables) that should be explicitly monitored. This is done to extract the required data which are essential for the adaptation process. The adaptation process in the proposed framework depends primarily on the following events: the new course enrolments, the completion of the learning style questionnaire and the completion of a self-assessment quiz. Therefore, the corresponding database tables are observed and monitored. This component is considered as a complementary part of the ERU in which events are recognised.

\section{- The Events Recognition Unit (ERU)}

This unit is responsible for detecting any changes that may occur to the tables identified in the OU process. The recognition process in the ERU can be based on any type of transaction in these tables such as INSERT, UPDATE and DELETE. However, in the proposed approach, INSERT transactions in specific tables can be seen as events in which the type of 
these events can be identified. For example, if a learner is enrolled in a particular course in the LMS, a record will be inserted in the table of enrolments. The new record indicates that a new learner has been enrolled in that course.

The ERU should differentiate between the feedback activity relevant to the learning styles and other feedback activities in the same course. In addition, the ERU should deal with different types of quiz and differentiate between them, especially the quizzes that are designed to provide feedback by email to the learners about their score.

From this point of view, the Rules Evaluation Unit (REU) (discussed in the following point) can play a major role in evaluating the conditions (rules) that are relevant to the aforementioned activities and crucial for the adaptation process. Course designers and teachers can identify pedagogical rules to control the adaptation process.

\section{- The Rules Evaluation Unit (REU)}

The REU is one of the most important parts in the ECA module. It performs the process of evaluating the pre-defined rules and conditions that can control the enforcement of the database triggers; the conditions and rules which are retrieved from the 'Shared' database are evaluated by the REU. However, if the conditions are satisfied, the required data about the events will be migrated to the "Shared" database, as Figure 1 shows. The activity diagram of the learning styles and self-assessment capturing process is depicted in Figure 4. 


\subsection{The Shared Database Schema Design}

The required events and associated data which meet the pre-defined pedagogical rules (discussed in Section 3.3) are stored in the "Shared" database using TCP/IP communication protocol. It is named "Shared" since it is shared between the ECA and the multi-agent modules, and the LMS. This process is considered to be the action part in the ECA module.

The "Shared" database is a normalised relational database that consists of the required database tables for the proposed adaptation process. The schema design of this database is shown in Figure 5. 


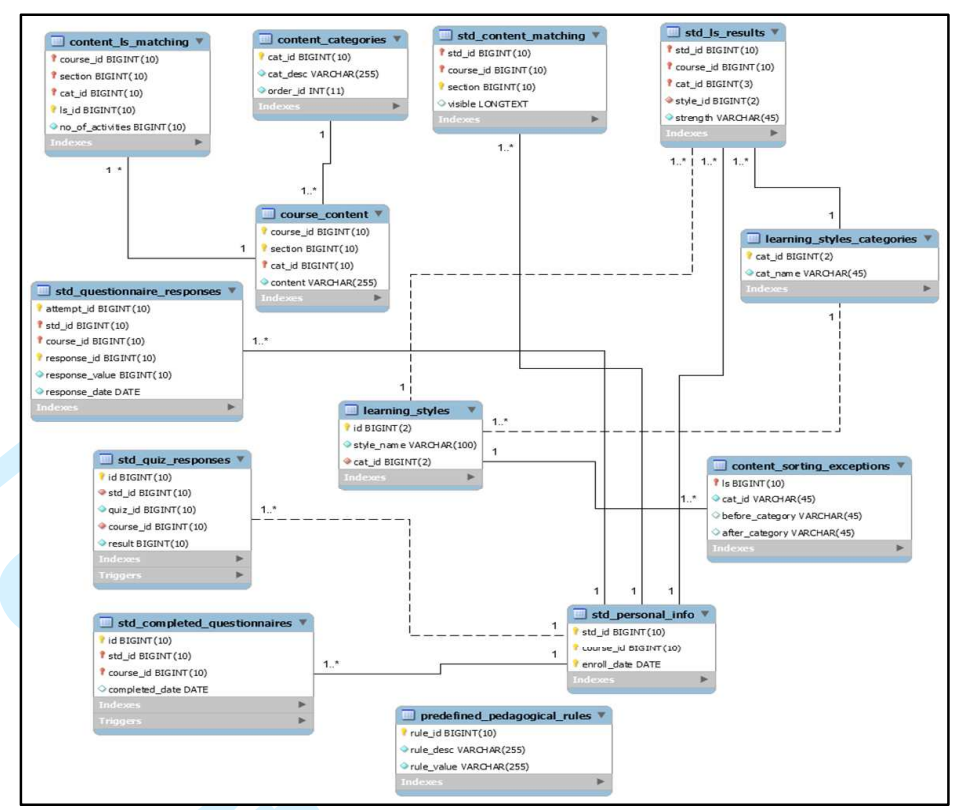

Figure 5: The Shared Database Schema Design

\subsection{The Multi-agent Module}

The multi-agent module is designed following the Gaia methodology (Wooldridge et al., 2000), which is a popular methodology for the analysis and design of agent-based systems. The multi-agent module consists of several agents, namely, the Control Agent (CA), the Learner Profile Agent (LPA), the Adaptive Agent (AA) and the Course Structure Agent (CSA). These agents work together to provide learners with adaptive contents based on their learning styles. The contents are presented using the Adaptive Interface Agent (AIA). The agents in the multi-agent module operate as follows:

\section{- The Control Agent}

The Control Agent is the first agent in the multi-agent module. It is responsible for detecting the relevant events in the learning styles questionnaire in the "Shared" database. Therefore, the $\mathrm{CA}$ is considered to be the backbone of the multi-agent module, and it controls the other agents in the system. It receives the required data about the events which are relevant to the learning styles questionnaire. These data are provided by the ECA module after fulfilling the pre-defined pedagogical rules. The CA informs the Learner Agent about these events and passes the corresponding data (ex. learner ID, course ID, event ID, and etc.) to the LA. The role model of the CA is summarised in Table 1. 


\section{- The Learner Agent}

The Learner Agent is responsible for collecting the required information about learners and their responses to the learning styles questionnaire. This process is based on the data sent by the CA. Furthermore, the LA has the role of classifying learners based on their preferred learning styles. It calculates the strength of the learning styles in the four dimension scale of the FSLSM. The learners are classified based on their preferences as Strong, Moderate, and Balanced in favour of a particular learning style in each of the four dimensions of the FSLSM.

The LA stores the data of the classification process in the 'Shared' database and provides the Adaptive Agent with the learning styles results of the learners. The role model of the LA is summarised in Table 2.

\section{- The Adaptive Agent}

The Adaptive Agent is responsible for the matching process between the content and learners' learning styles based on the classification results. Based on these results, the AA can match the course contents with the learners' learning styles. The matching process is based on the proposed adaptive features which have been discussed previously in this paper. Furthermore, the AA communicates with the Course Structure Agent to request the course contents that meet the learning styles' needs. Consequently, the CSA provides the AA with the contents appropriate to the learner's learning styles. Once these contents are received, the AA applies the adaptive features to these contents and stores the adaptive contents for each learner into the 'Shared' database. This process is based on the proposed adaptive features taking into consideration the weights of the learning style strengths; the weights for Strong, Moderate and Balanced are (3), (2) and (1) respectively. These weights are considered in the adaptation process in order to decide the number of learning resources and activities and deal with any conflicts between learning styles.

The AA is responsible for ordering the contents for the learners based on any criteria which can be identified by the course designers and teachers. The ordering process is designed to be a dynamic process in which the AA can follow any ordering criteria. The role model of AA is summarised in Table 3. 
- The Course Structure Agent

The Course Structure Agent deals with the structure of the course in the LMS. It collects the learning resources and activities that can support and affect the learning styles of the learners. This information is crucial for the AA in order to complete the adaptation process. The role model of the CSA is summarised in Table 4.

Each agent in the multi-agent module is instantiated once and has as many instances as the number of the events received. The agent model of the multi-agent module is depicted in Figure 6.

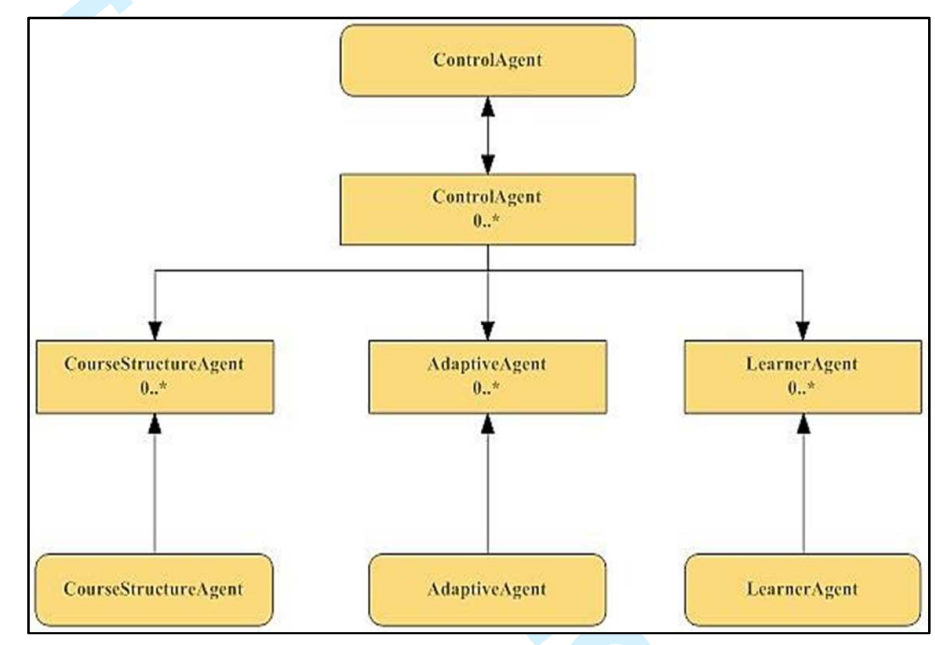

Figure 6: The agent model of the multi-agent module

The service model in Gaia aims to identify the services which are relevant to each agent role along with their properties. The properties of these services can be identified in terms of inputs, outputs, pre-conditions and post-conditions. The inputs and outputs properties can be derived from the activities and protocols which have already been identified in the role model. On the other hand, the pre-conditions and post-conditions can be identified from the safety property of the role. The main services in the multi-agent module are summarized in Table 5.

The multi-agent module has four agents which communicate with each other in order to provide learners with adaptive contents based on their preferred learning styles. The acquaintance model of the multi-agent module is shown in Figure 7. 


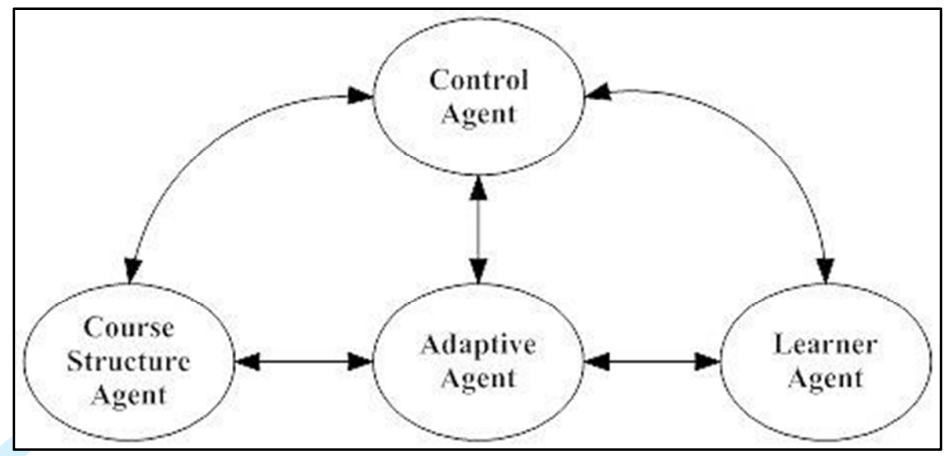

Figure 7: The acquaintance model of the multi-agent module

The multi-agent module is developed using JADE (Bellifemine et al., 2007). The agents work together in order to provide learners with adaptive contents in the LMS. This process is supported by the ECA module which is responsible for providing the multi-agent module with the required events in a timely manner. Figure 8 shows our system prototype of the multi-agent module in JADE.

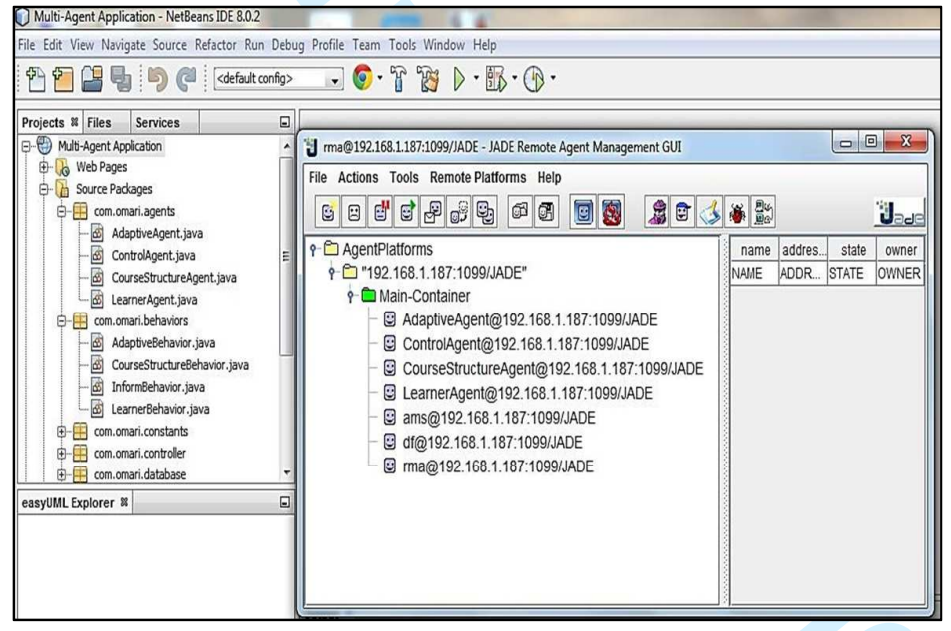

Figure 8: The multi-agent module in JADE

Once the learner submits the questionnaire in the LMS, the agents cooperate in a timely manner and provide the learner with adaptive contents that suit his/her learning styles via the adaptive interface agent, which follows Moodle interface standards. The interaction between the agents when a learner completes the learning styles questionnaire is shown in Figure 9. 


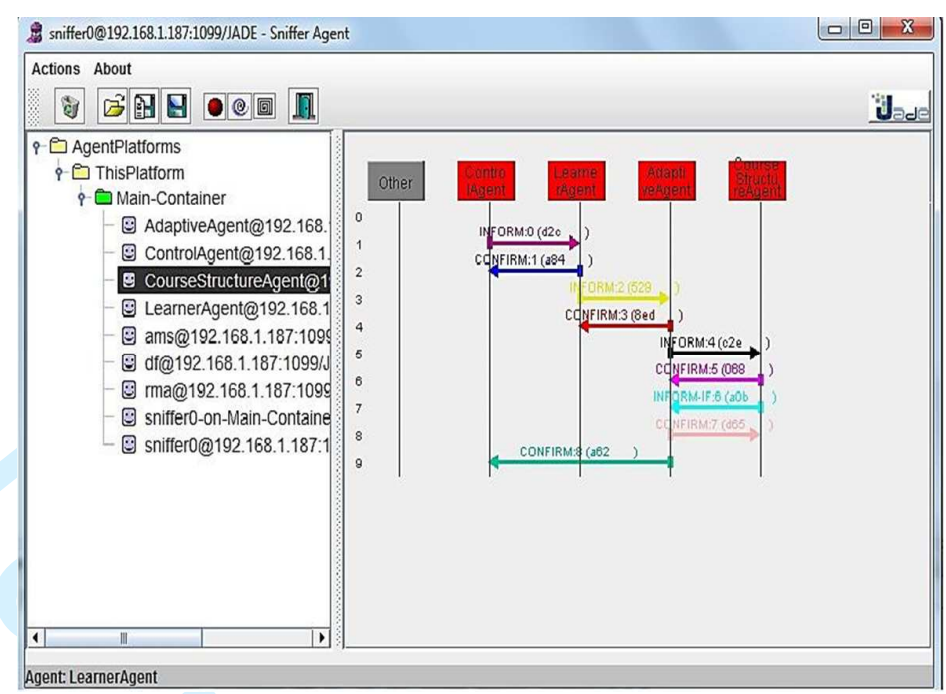

Figure 9: The interaction of agents in JADE

The final objective of this interaction is to provide the learner with adaptive contents based on the results of the learning styles questionnaire. Figure 10 shows an example of an adaptive course for a particular learner. The adaptive features include the type, number, and order of learning activities and resources. These features can be easily modified by teachers and course designers via the GUI. The proposed approach can deal with any type of contents that can be presented in the LMS. Besides this, it supports adaptivity in a timely manner at the course level.

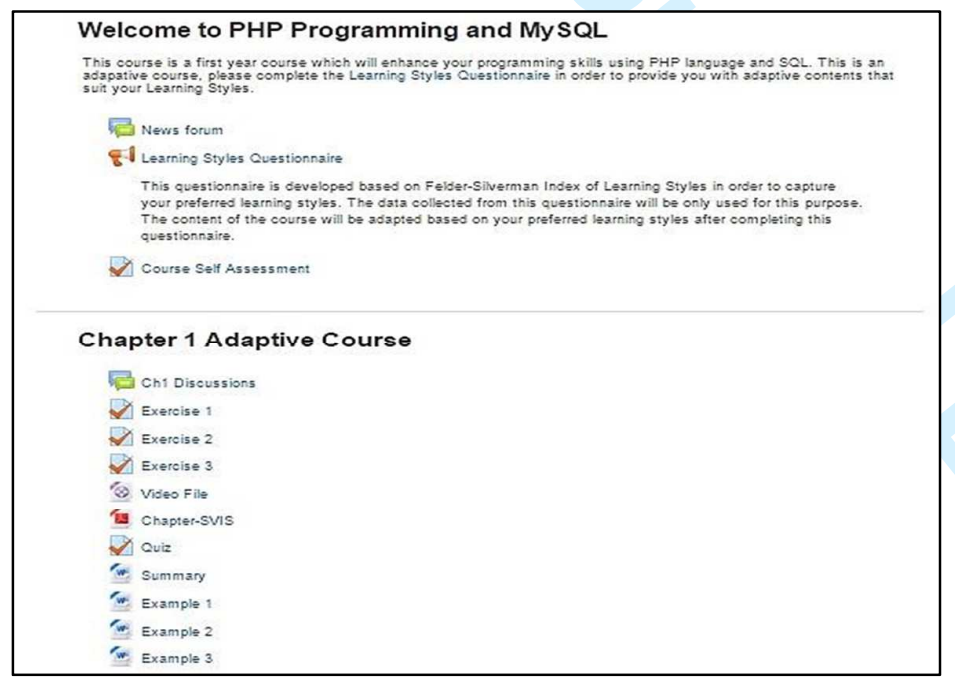

Figure 10: Adaptive course for a particular learner 


\subsection{Discussion}

In the previous sections, we discussed our framework in detail, highlighting a novel hybrid approach using agent technology and the concept of the ECA model. We also presented a system prototype that reflects the design of the framework. We claim that the proposed framework can be applied in any LMS to support adaptivity based on learning styles. Moreover, it provides real-time adaptivity using the technologies introduced earlier in this paper. It can deal with any type of material that can be presented in the LMS, providing a dynamic, adaptive experience that can be controlled based on pre-defined criteria. As stated earlier in this paper, no work has been published incorporating the technologies mentioned in this paper. We proposed a novel hybrid approach to support real-time adaptivity in LMSs in order to enhance and improve the learning process in these systems.

The work presented in this paper suggests that adaptivity can be technically achieved using agent technology and the concept of the ECA model in the context of e-learning. This could point towards further research on using agent technology in the context of e-learning. For example, the proposed framework can be adapted to support other adaptive experiences, based on learners' knowledge level and disabilities, for instance.

\section{Conclusion and Future Work}

Learning Management Systems are vital for successful adoption of distance and blended learning. Most of these systems take a one-size-fits-all approach. In this paper, a proposed framework reflecting a hybrid approach is designed to support adaptivity in any given Learning Management System. A system prototype is developed using the concept of the ECA model and intelligent agents to support adaptivity based on learning styles. This paper presents the design and prototype of the proposed approach. The prototype is validated in Moodle. It shows that the proposed hybrid approach is technically feasible. We believe that our approach can be implemented in any e-learning environment, including MOOCs. It depends on implementing the required database triggers in the database of the LMS. Moreover, the proposed approach supports adaptivity in a timely manner and could incorporate a dynamic adaptive process. In future, we intend to evaluate our approach as a case study in Moodle following different live scenarios. 


\section{References}

Al-Azawei, A. and Badii, A. (2014), "STATE OF THE ART OF LEARNING STYLESBASED ADAPTIVE EDUCATIONAL HYPERMEDIA SYSTEMS (LS-BAEHSS)", Int. J. Comput. Sci. Inf. Technol, Vol. 6 No. 3, pp. 1-19.

Al-Omari, M., Carter, J. and Chiclana, F. (2015), "A Proposed Framework to Support Adaptivity in Virtual Learning Environments", Proceedings of The European Conference on Technology in the Classroom 2015, Brighton, United Kingdom, The International Academic Forum (IAFOR), pp. 257-264.

Bellifemine, F., Caire, G. and Greenwood, D. (2007), Developing Multi-Agent Systems with $J A D E$, John Wiley \& Sons Ltd, England.

Bokhari, M.U. and Ahmad, S. (2014), "Multi-Agent Based E-Learning Systems: A Comparative Study", Proceedings of the 2014 International Conference on Information and Communication Technology for Competitive Strategies, ACM, p. 20.

Bra, P. De, Aerts, A., Berden, B., Lange, B. de, Rousseau, B., Santic, T., Smits, D., et al. (2003), “AHA! The adaptive hypermedia architecture", Proceedings of the fourteenth ACM conference on Hypertext and hypermedia - HYPERTEXT ’03, Vol. 4, p. 81.

Brusilovsky, P. (2004), “KnowledgeTree: A distributed architecture for adaptive e-learning”, Proceedings of the 13th international World Wide Web conference on Alternate track papers \& posters, ACM, pp. 104-113.

Carver, C.A., Howard, R.A. and Lane, W.D. (1999), “Addressing different learning styles through course hypermedia", IEEE Transactions on Education, Vol. 42 No. 1, pp. 3338.

Cha, H.J., Kim, Y.S., Park, S.H., Yoon, T.B., Jung, Y.M. and Lee, J.-H. (2006), "Learning styles diagnosis based on user interface behaviors for the customization of learning interfaces in an intelligent tutoring system”, Intelligent tutoring systems, Springer, pp. $513-524$.

Chang, Y.-H. and Chen, Y.-Y. (2012), “A mashup-based adaptive learning system”, International Conference on Machine Learning and Cybernetics (ICMLC), IEEE, Xian, Vol. 5, pp. 1721-1726. 
Dunn, R. and Griggs, S. (2003), "Synthesis of the Dunn and Dunn learning styles model research: who, what, when, where and so what-the Dunn and Dunn learning styles model and its theoretical cornerstone", St. John's University, New York.

Felder, R.M. and Silverman, L.K. (1988), "Learning and Teaching Styles in Engineering Education”, Engineering Education, Vol. 78 No. 7, pp. 674-681.

Felder, R.M. and Soloman, B.A. (2005), "Index of learning styles questionnaire”, available at: http://www.engr.ncsu.edu/learningstyles/ilsweb.html (accessed 1 March 2015).

Gilbert, J.E. and Han, C.Y. (1999), "Arthur: An adaptive instruction system based on learning styles”, International Conference on Mathematics/Science Education and Technology, Vol. 1999, pp. 100-105.

Graf, S. (2007), “Adaptivity in learning management systems focussing on learning styles”, Vienna University of Technology.

Honey, P. and Mumford, A. (1992), The manual of learning styles, Peter Honey Maidenhead, 3rded.

Huang, E.Y., Lin, S.W. and Huang, T.K. (2012), "What type of learning style leads to online participation in the mixed-mode e-learning environment? A study of software usage instruction", Computers \& Education, Elsevier, Vol. 58 No. 1, pp. 338-349.

Kolb, A.Y. (2005), "The Kolb learning style inventory-version 3.12005 technical specifications", Boston, MA: Hay Resource Direct, Vol. 200.

Kolb, D.A. (1984), Experiential Learning: Experience as the Source of Learning and Development, Prentice-Hall, Englewood Cliffs, New Jersey.

Litzinger, T.A., Lee, S.H., Wise, J.C. and Felder, R.M. (2007), “A psychometric study of the index of learning styles(’", Journal of Engineering Education, Wiley Online Library, Vol. 96 No. 4, pp. 309-319.

Moodle. (2016), "Moodle Open source Learning platform”, available at: https://moodle.org/ (accessed 14 March 2016).

Morales-Rodríguez, M.L., Ramírez-Saldivar, J.A., Sánchez-Solís, J.P. and HernándezRamírez, A. (2012), “Design of an Intelligent Agent for Personalization of Moodle's 
Contents", Research in Computing Science, Vol. 56, pp. 11-17.

Papanikolaou, K.A., Grigoriadou, M., Kornilakis, H. and Magoulas, G.D. (2003), "Personalizing the Interaction in a Web-based Educational Hypermedia System: the case of INSPIRE", User modeling and user-adapted interaction, Springer, Vol. 13 No. 3, pp. 213-267.

Phobun, P. and Vicheanpanya, J. (2010), “Adaptive intelligent tutoring systems for e-learning systems", Procedia-Social and Behavioral Sciences, Elsevier, Vol. 2 No. 2, pp. 40644069.

Pitigala Liyanage, M.P., Gunawardena, K.S.L. and Hirakawa, M. (2013), “A framework for adaptive learning management systems using learning styles”, 2013 International Conference on Advances in ICT for Emerging Regions (ICTer), IEEE, Colombo, pp. 261-265.

Poulovassilis, A., Papamarkos, G. and Wood, P. (2006), "Event-condition-action rule languages for the semantic web", In Current Trends in Database Technology-EDBT, Springer Berlin Heidelberg, pp. 855-864.

Riding, R.J. (1991), “Cognitive styles analysis", Learning and Training Technology, Birmingham.

Shang, Y., Shi, H. and Chen, S.-S. (2001), “An intelligent distributed environment for active learning", Journal on Educational Resources in Computing (JERIC), ACM, Vol. 1 No. 2es, p. 4.

Villaverde, J.E., Godoy, D. and Amandi, A. (2006), "Learning styles' recognition in elearning environments with feed-forward neural networks", Journal of Computer Assisted Learning, Wiley Online Library, Vol. 22 No. 3, pp. 197-206.

Viola, S.R., Graf, S. and Leo, T. (2006), “Analysis of Felder-Silverman index of learning styles by a data-driven statistical approach", Multimedia, 2006. ISM'06. Eighth IEEE International Symposium on, IEEE, pp. 959-964.

Wolf, C. (2003), “iWeaver: towards' learning style'-based e-learning in computer science education", Proceedings of the fifth Australasian conference on Computing educationVolume 20, Australian Computer Society, Inc., pp. 273-279. 
Wooldridge, M., Jennings, N.R. and Kinny, D. (2000), “The Gaia methodology for agentoriented analysis and design", Autonomous Agents and multi-agent systems, Springer, Vol. 3 No. 3, pp. 285-312.

Xu, D., Huang, W.W., Wang, H. and Heales, J. (2014), "Enhancing e-learning effectiveness using an intelligent agent-supported personalized virtual learning environment: An empirical investigation”, Information \& Management, Elsevier B.V., Vol. 51 No. 4, pp. $430-440$.

Yang, T.-C., Hwang, G.-J. and Yang, S.J.-H. (2013), "Development of an Adaptive Learning System with Multiple Perspectives based on Students? Learning Styles and Cognitive Styles.”, Educational Technology \& Society, Vol. 16 No. 4, pp. 185-200.

Zhang, C., Zhao, R. and Zhou, Z. (2010), “An Agent-based Architecture for E-Learning System", The 2nd International Workshop on Intelligent Systems and Applications (ISA), IEEE, pp. 1-4.

Zhi-xue, W., Xin, J., Qing-chao, D., Hong-yue, H. and Qing-long, W. (2012), "ECA rule modeling language based on UML", International Conference on Computer Science and Automation Engineering (CSAE), IEEE, Vol. 1, pp. 623-628. 
Table 1: The role model of the CA

\begin{tabular}{|c|c|}
\hline Role: & ControlAgent (CA) \\
\hline Description: & $\begin{array}{l}\text { This agent is responsible for receiving the required data which are } \\
\text { relevant to the learning styles questionnaire events such as the learner ID, } \\
\text { course ID and event ID. It informs the Learner Agent that these events } \\
\text { have been detected and provides the LA with the data associated with } \\
\text { these events. }\end{array}$ \\
\hline $\begin{array}{l}\text { Protocols \& } \\
\text { Activities: }\end{array}$ & DetectEvent,InformLearnerAgent,SendEventData \\
\hline Permissions: & Reads events data \\
\hline Responsibilities: & $\begin{array}{l}\text { Liveness: CONTROLAGENT }= \\
\text { (DetectEvent.InformLearnerAgent.SendEventData) })_{\omega^{*}} \\
\text { Safety: Database triggers are activated in the LMS. } \\
\text { The Shared database is accessible and running }\end{array}$ \\
\hline
\end{tabular}

Table 2: The role model of the $\mathrm{LA}$

\begin{tabular}{|ll|}
\hline Role: & LearnerAgent (LA) \\
\hline Description: & $\begin{array}{l}\text { This agent is responsible for classifying learners based on their preferred } \\
\text { learning styles. It collects the required information about learners and } \\
\text { their responses to the learning styles questionnaire based on the data sent } \\
\text { by the CA. It provides the Adaptive Agent with the results of the } \\
\text { classification and stores these results in the Shared database. }\end{array}$ \\
\hline $\begin{array}{l}\text { Protocols \& } \\
\text { Activities: }\end{array}$ & $\begin{array}{l}\text { ReceiveInformMessage,GetLearnerInfo,CalculateLearningStyles, } \\
\text { ClassifyLearners,StoreClassResults,SendResults }\end{array}$ \\
\hline Permissions: & $\begin{array}{l}\text { Reads from the Shared database } \\
\text { Writes to the Shared database }\end{array}$ \\
\hline Responsibilities: & $\begin{array}{l}\text { Liveness: LEARNERAGENT = } \\
\text { (ReceiveInformMessage.GetLearnerInfo. } \\
\text { CalculateLearningStyles.ClassifyLearners.StoreClassResults.SendResult } \\
\text { s) })\end{array}$ \\
& Safety: The Shared database is accessible and running. \\
\hline
\end{tabular}

Table 3: The role model of AA

\begin{tabular}{|ll|}
\hline Role: & AdaptiveAgent (AA) \\
\hline Description: & This agent is responsible for the matching process between the content \\
& and learners' learning styles based on the classification results. Also, it \\
& has the role of ordering the content which are received from the CA and \\
& deciding the number of content to be shown for each learner. It applies \\
& the proposed adaptive features of the system. After the adaptation process \\
& is completed, the AA stores the adaptive content for each learner in the \\
\hline
\end{tabular}


Shared database and confirms whether the mission is completed successfully or not.

Protocols \& ReceiveClassResults, RequestCourseContent,MatchLSContents, Activities: CalculateNoActivites,ContentsOrdering,CheckOrderingExceptions, StoreAdaptiveContents,SendConfirmation

Permissions: $\quad$ Reads from the Shared database

Responsibilities: Writes to the Shared database

Liveness: ADAPTIVEAGENT $=($ ReceiveClassResults.

RequestCourseContent.MatchLSContents. CalculateNoActivites. ContentsOrdering.CheckOrderingExceptions.StoreAdaptiveContents. SendConfirmation) $\omega$

Safety: The Shared database is accessible and running.

Table 4: The role model of the CSA

\begin{tabular}{|ll|}
\hline Role: & CourseStructureAgent (CSA) \\
\hline Description: & $\begin{array}{l}\text { This agent is responsible for providing the AA with the course structure } \\
\text { including the learning resources and activities that can support and affect } \\
\text { the learning styles of the learners. }\end{array}$ \\
\hline $\begin{array}{l}\text { Protocols \& } \\
\text { Activities: }\end{array}$ & $\begin{array}{l}\text { ReceiveClassResults,SendReceiveConfirmation,GetCourseContents, } \\
\text { SendContents, ReceiveConfirmation }\end{array}$ \\
\hline Permissions: & Reads from the Shared database \\
\hline Responsibilities: & $\begin{array}{l}\text { Liveness: COURSESTRUCTUREAGENT = (ReceiveClassResults. } \\
\text { SendReceiveConfirmation.GetCourseContents. SendContents. } \\
\text { ReceiveConfirmation) } \omega \\
\\
\text { Safety: The Shared database is accessible and running. }\end{array}$ \\
\hline
\end{tabular}

Table 5: The main services in the multi-agent module

\begin{tabular}{|l|l|}
\hline Service & Detect learning styles questionnaire event. \\
\hline Inputs & Text file \\
\hline Outputs & Data about the event (ex. Event ID, learner ID and course ID) \\
\hline Pre-condition & $\begin{array}{l}\text { The multi-agent module is instantiated and running, and the ECA } \\
\text { module is activated in the LMS. The learner submits the learning } \\
\text { styles questionnaire. }\end{array}$ \\
\hline Post-condition & - \\
\hline Service & Obtain learning styles questionnaire responses \\
\hline Inputs & Data about the event (ex. Event ID, learner ID and course ID) \\
\hline Outputs & $\begin{array}{l}\text { Learning styles results by classifying the learner based on his/her } \\
\text { preferred learning styles }\end{array}$ \\
\hline Pre-condition & The multi-agent module is instantiated and running. \\
\hline
\end{tabular}




\begin{tabular}{|l|l|}
\hline Post-condition & The Shared database is accessible using a communication protocol. \\
\hline Service & Obtain course content \\
\hline Inputs & Course ID and the preferred learning styles \\
\hline Outputs & The course structure that meets the learning styles preference \\
\hline Pre-condition & The multi-agent module is instantiated and running. \\
\hline Post-condition & The Shared database is accessible using a communication protocol. \\
\hline Service & Adapt content \\
\hline Inputs & Learning styles results, course structure and content \\
\hline Outputs & $\begin{array}{l}\text { Adaptive content based on learner's preferred learning styles } \\
\text { incorporating the proposed adaptive features }\end{array}$ \\
\hline Pre-condition & The multi-agent module is instantiated and running. \\
\hline Post-condition & The Shared database is accessible using a communication protocol. \\
\hline
\end{tabular}

